\title{
Functional Neurosurgery in Africa: A Scoping Review Protocol
}

Authors: Nathalie Christelle Ghomsi ${ }^{1,2}$ Aminata Yandeh Sallah ${ }^{1,3}$, Kantenga Dieu Merci Kabulo $^{1,3}$, Ulrick Sidney Kanmounye ${ }^{1,5}$, Crescencia Mashauri ${ }^{1,7}$, Stephane Nguembu ${ }^{1,6}$, Abdullahi Jimoh ${ }^{4}$,

\section{Institutional affiliations:}

1. Research Department, Association of Future African Neurosurgeons, Yaounde, Cameroon

2. Neurosurgery Unit, Department of Surgery, Felix-Houphouet Boigny University, Abidjan, Côte d'Ivoire

3. Department of Neurosurgery, University of Zimbabwe, Harare, Zimbabwe

4. Division of Neurosurgery, Department of Surgery, Ahmadu Bello University Teaching Hospital, Shika Zaria, Nigeria

5. Faculty of Medicine, Bel Campus University of Technology, Kinshasa, DR Congo

6. Higher Institute of Health Sciences Université des Montagnes, Bangante, Cameroon

7. Medical institute, Tambov state university, Tambov, Russia 


\section{BACKGROUND AND SIGNIFICANCE}

Functional neurosurgery (FN) is a set of procedures that involves precise surgical targeting of anatomic structures to modulate or improve a neurologic function (1). The end of the 19th century marked the inception of functional neurosurgery, by Horsley and colleagues as they performed corticectomies for athetosis and chorea (2). During the 20th and 21 st centuries, the neurosurgical field focalized on accurately targeted surgery, thus improving the functional outcome of neurosurgically operated or cancer patients. FN has become a crucial tool in managing diseases such as intractable epilepsy, pain, neurodegenerative disorders, and psychiatric disorders. Such conditions require a multidisciplinary approach (3). Moreover, the advent of new imaging modalities, neuronavigation, and stereotactic surgery has expanded and developed targeted treatment modalities such as pallidotomy, Deep Brain Stimulation (DBS), and rhizotomy. These techniques provide a safe corridor to deep-seated lesions and better functional outcomes (4). The ergonomics of these devices are still not optimal to date, so innovations are necessary (12).

The prevalence of $\mathrm{FN}$ diseases is higher in developing countries than in developed countries, particularly in Africa (5). These diseases are a barrier to the attainment of at least six sustainable development goals. For example, they increase poverty and hunger because they result in poor functional prognosis when treatment is delayed or inadequate (6). FN's handicap reduces productivity, workforce, time spent in education, and exacerbates gender inequality since women are often the primary caretakers (6).

In Africa, few centers offer FN, despite the increasing incidence of $\mathrm{FN}$ diseases. Besides aspiring to FN modalities, most developing countries still lack an adequate number of medical workforce, neurosurgical facilities, and neurosurgical training. Existing FN centers face multiple challenges. The most important being the lack of equipment due to its prohibitive costs and the lack of procurement funds. The shortage of neurosurgical 
facilities includes dedicated neurosurgical beds, intensive care unit beds, and hospitals with adequate neurosurgery capacity. There is also an identical problem with neurosurgeons concentrated in the urban compared to suburban areas with a high discrepancy. As a result, privileged African patients resort to medical tourism for their care (7). The dearth of specialized neurosurgeons to ensure accurate diagnosis and treatment exacerbates the ongoing situation resulting in an increased mortality rate $(8,13)$. Furthermore, the poor cooperation between neurosurgical societies, low allocation, and poor development in neurosurgery education programs by the governing bodies in the developing countries have been identified as the main factors for the slow improvement in the neurosurgery services within those countries (9). Fezeu et al. described the different challenges faced by low- and middle-income countries (LMICs) in the practice of functional neurosurgery and proposed some solutions (10). Some of the solutions include context-specific procedure standardization (10), aberration between high-income countries (HICs), and LMICs such as the Tunisian-French collaboration in 2006 (11) on intractable epilepsy surgery, and equipment donations.

\section{STUDY RATIONALE}

The scarcity of data on the FN practice in Africa justifies a scoping review to map the availability of FN resources and its surgical volume. At the end of this study, we hope to highlight areas with a high disease burden and little or no access to FN care and to spur research access to FN in Africa.

\section{STUDY OBJECTIVE}

This scoping review aims to map out FN activity in Africa. 


\section{METHODS}

\section{A. Study Design}

We will use a scoping review methodology to ensure broad research areas on the subject and study designs. We will use Arksey and O'Malley's scoping review methodology:

identify the research question; (2) identify relevant studies; (3) select studies; (4) extract data from the reviews; and (5) illustrate the data.

Stage 1: Identify the research question

After consultation with African neurosurgeons and patients, we developed the following research questions:

1. Where are functional neurosurgery procedures done in Africa?

2. Which functional neurosurgery procedures are performed in Africa?

3. Why are functional neurosurgery procedures done in Africa? (diseases, indications)

4. Which patients get functional neurosurgery in Africa?

5. What is the surgical volume of functional neurosurgery in Africa?

6. What are the barriers to functional neurosurgery in Africa?

Stage 2: Identify relevant studies

PubMed/MEDLINE, EMBASE, African Journals Online, LILACS, WHOLIS, Google Scholar, and Web of Science will be searched from inception. No language limit will be applied. Based on the initial exploratory research, we have agreed on the eligibility criteria:

Publication type journal articles, abstracts, and case reports

Time frame: any 
Language: any

- Types of interventions: all functional neurosurgery interventions carried out in Africa

Exclusion criteria

* All non-functional neurosurgery studies

Studies with neither full-texts nor abstracts and articles without full-texts after the abstract and title review

* Studies in which the setting is non-African

The key terms we will use are: "Functional Neurosurgery" AND " Interventions" AND "Africa." Articles will be retrieved from each database and imported into a free online reference management software - Rayyan.

\section{Stage 3: Study selection}

The team will upload the search results to Rayyan and proceed with deduplication. All team members will then screen titles and abstracts to exclude those that do not meet the eligibility criteria identified in the second stage of the protocol. Each article will be screened independently twice. Conflicts will be resolved first by the two reviewers, but a third reviewer will serve as an arbitrator if the two cannot agree.

The retrieved articles and those with uncertain eligibility criteria will be screened by another team member to ensure consistent inclusion criteria in the review. 


\section{Stage 4: Data collection}

Extraction of study characteristics and relevance will be done using a standardized data collection instrument - hosted on Google Forms. The extracted data will include authors, publication year, publication type, study design, country, surgical technique, disease, modality used, and difficulties. A review and pretesting of the form will be carried out before application to ensure accurate data registering. For accuracy, each reviewer's independent extracted data will be further discussed to guarantee coherence between reviewers.

Stage 5: Illustrating the data

A narrative description of the search decision process will be mapped on a flowchart. This flowchart will detail the review decision process, indicate the search results, excluded duplicate citations, study selection criteria, full retrieval, and additions from reference list searching and final summary presentation.

A narrative description of the findings will be done and the resulting manuscript will be published in a peer-reviewed journal. The abstract will be submitted to international conferences and visual abstracts will be used for social media dissemination. 


\section{REFERENCES}

1. Speelman JD, Bosch DA. Resurgence of functional neurosurgery for Parkinson's disease: A historical perspective. Mov Disord. 1998;13(3):582-8.

2. Horsley V. The Linacre Lecture ON THE FUNCTION OF THE SO-CALLED MOTOR AREA OF THE BRAIN. Br Med J. 1909 Jul 17;2(2533):121-32.

3. Kanpolat $Y$. Percutaneous destructive pain procedures on the upper spinal cord and brain stem in cancer pain: CT-guided techniques, indications and results. Adv Tech Stand Neurosurg. 2007;147-73.

4. Benabid AL, Chabardes S, Torres N, Piallat B, Krack P, Fraix V, et al. Functional neurosurgery for movement disorders: a historical perspective. Prog Brain Res. 2009;175:379-91.

5. Diop AG, Hesdorffer DC, Logroscino G, Hauser WA. Epilepsy and Mortality in Africa: A Review of Literature. Epilepsia. 2005;46(s11):33-5.

6. Rosa W, editor. Transforming Our World: The 2030 Agenda for Sustainable Development. In: A New Era in Global Health [Internet]. New York, NY: Springer Publishing Company; 2017 [cited 2020 Sep 25]. Available from: http://connect.springerpub.com/lookup/doi/10.1891/9780826190123.ap02

7. WHO | Evidence on global medical travel [Internet]. WHO. World Health Organization; [cited 2020 Sep 20]. Available from: http://www.who.int/bulletin/volumes/93/11/14-146027/en/

8. Dewan MC, Rattani A, Gupta S, Baticulon RE, Hung Y-C, Punchak M, et al. Estimating the global incidence of traumatic brain injury. J Neurosurg. 2018;130(4):1080-1097.

9. Kato Y, Liew BS, Sufianov AA, Rasulic L, Arnautovic KI, Dong VH, et al. Review of global neurosurgery education: Horizon of Neurosurgery in the Developing Countries. Chinese Neurosurgical Journal [Internet]. 2020 Dec [cited 2020 Oct 2];6(1).

10. Fezeu F, Ramesh A, Melmer PD, Moosa S, Larson PS, Henderson F. Challenges and Solutions for Functional Neurosurgery in Developing Countries. Cureus [Internet]. [cited 2020 Jul 20];10(9). 
11. Mrabet Khiari H, Khemiri E, Parain D, Hattab N, Proust F, Mrabet A. Epilepsy surgery program in Tunisia: An example of a Tunisian French collaboration. Seizure. 2010 Mar 1;19(2):74-8.

12. Kockro RA, Tsai YT, Ng I, Hwang P, Zhu C, Agusanto K, Hong LX, Serra L (2009) Dex-ray: augmented reality neurosurgical navigation with a handheld video probe. Neurosurgery 65:795-807; discussion 807-798.

13. Karekezi C, El Khamlichi A, El Ouahabi A, El Abbadi N, Ahokpossi SA, Ahanogbe $\mathrm{KMH}$, et al. The impact of African-trained neurosurgeons on sub-Saharan Africa. Neurosurg Focus. 2020 01;48(3):E4. 


\section{APPENDICES [1]}

\section{Search Strategy (Detailed PubMed search strategy)}

"Electric stimulation therapy"[MeSH Terms] OR Electric Stimulation Therapy[Text Word] OR Therapeutic Electrical Stimulation OR Electrical Stimulation, Therapeutic OR Stimulation, Therapeutic Electrical OR Therapeutic Electric Stimulation OR Electric Stimulation, Therapeutic OR Stimulation, Therapeutic Electric OR Electrical Stimulation Therapy OR Stimulation Therapy, Electrical OR Therapy, Electrical Stimulation OR Therapy, Electric Stimulation OR Stimulation Therapy, Electric OR Electrotherapy OR Interferential Current Electrotherapy OR Electrotherapy, Interferential Current OR Brain Stimulations, Deep OR Deep Brain Stimulations OR Stimulation, Deep Brain OR Stimulations, Deep Brain OR Brain Stimulation, Deep OR Electrical Stimulation of the Brain AND Cord Stimulation, Spinal OR Stimulation, Spinal Cord AND Cordotomies OR Chordotomy OR Chordotomies AND "rhizotomy"[MeSH Terms] OR rhizotomy[Text Word]Rhizotomies AND Psychosurgeries OR Lobotomy OR Lobotomies OR Gyrectomy OR Gyrectomies OR Leukotomy OR Leukotomies OR Leucotomy OR Leucotomies OR Topectomy OR Topectomies AND Stereotaxic Technique OR Technique, Stereotaxic OR Techniques, Stereotaxic OR Stereotactic Techniques OR Stereotactic Technique OR Technique, Stereotactic OR Techniques, Stereotactic OR Stereotaxic Technics OR Stereotaxic Technic OR Technic, Stereotaxic OR Techniques, Stereotaxic AND Denervation OR Denervations OR Laser Neurectomy OR Laser Neurectomies OR Neurectomies, Laser OR Neurectomy, Laser OR Radiofrequency Neurotomy OR Neurotomies, Radiofrequency OR Neurotomy, Radiofrequency OR Radiofrequency Neurotomies OR Neurectomy OR Neurectomies OR Peripheral Neurectomy OR Neurectomies, Peripheral OR Neurectomy, Peripheral OR Peripheral Neurectomies AND Pallidotomy OR Pallidotomies AND Denervation OR Denervation, Autonomic OR Autonomic Denervations OR Denervations, Autonomic AND Parasympathectomy OR Parasympathectomies OR Parasympathetic Denervation OR Denervation, Parasympathetic OR Denervations, Parasympathetic OR Parasympathetic Denervations AND [Movement Disorder OR Movement Disorder Syndromes OR Movement Disorder Syndrome OR Dyskinesia Syndromes OR Dyskinesia Syndrome OR Etat Marbre OR Status Marmoratus OR Developmental Psychomotor Disorders OR Developmental Psychomotor Disorder OR Psychomotor Disorder, Developmental OR Psychomotor Disorders, Developmental OR Psychomotor Impairment OR Impairment, Psychomotor OR Impairments, Psychomotor OR Psychomotor Impairments AND Mental Disorder OR Psychiatric Diseases OR Psychiatric Disease OR Psychiatric Illness OR Psychiatric Illnesses OR Psychiatric Disorders OR Psychiatric Disorder OR Diagnosis, Psychiatric OR Psychiatric Diagnosis OR Behavior Disorders OR Mental Disorders, Severe Mental Disorder, Severe OR Severe Mental Disorder OR Severe Mental Disorders AND "Obsessive-compulsive disorder" OR Disorder, Obsessive-Compulsive OR Disorders, 
Obsessive-Compulsive OR Obsessive Compulsive Disorder OR Obsessive-Compulsive Disorders OR Neurosis, Obsessive-Compulsive OR Neuroses, Obsessive-Compulsive OR Neurosis, Obsessive Compulsive OR Obsessive-Compulsive Neuroses OR Obsessive-Compulsive Neurosis OR Anankastic Personality OR Anankastic Personalities OR Personalities, Anankastic OR Personality, Anankastic AND Mood disorder OR Disorder, Mood OR Disorders, Mood OR Mood Disorder OR Affective Disorders OR Affective Disorder OR Disorder, Affective OR Disorders, Affective] AND [Africa, Central OR Central Africa AND Cameroon OR Republic of Cameroon OR United Republic of Cameroon OR Cameroons AND Central African Republic OR Ubangi-Shari AND Chad AND Congo OR Republic of the Congo OR Congo (Brazzaville) AND Democratic Republic of the Congo OR Congo (Kinshasa) OR Zaire OR Belgian Congo OR Katanga AND Equatorial Guinea OR Republic of Equatorial Guinea OR Spanish Guinea OR Guinea, Spanish OR Rio Muni AND Gabon OR Gabonese Republic AND Sao Tome and Principe AND Africa, Eastern OR East Africa OR Eastern Africa OR British Indian Ocean Territory AND Burundi OR Republic of Burundi OR Urundi AND Djibouti OR Somaliland, French OR Republic of Djibouti OR French Somaliland AND Eritrea OR Ethiopia OR Federal Democratic Republic of Ethiopia AND Kenya OR Republic of Kenya AND Rwanda OR Republic of Rwanda OR Ruanda AND Somalia AND South Sudan OR Sudan OR Republic of the Sudan AND Tanzania OR United Republic of Tanzania OR Zanzibar OR Tanganyika AND Uganda OR Republic of Uganda AND Africa, Southern OR Southern Africa AND Angola AND Botswana OR Bechuanaland OR Kalahari AND Eswatini OR Swaziland AND Lesotho OR Basutoland OR Kingdom of Lesotho AND Malawi OR Republic of Malawi OR Nyasaland AND Mozambique OR Republic of Mozambique OR Portuguese East Africa AND Namibia OR Southwest Africa OR Republic of Namibia OR South West Africa AND South Africa OR Union of South Africa OR Republic of South Africa AND Zambia OR Rhodesia, Northern OR Northern Rhodesia OR Republic of Zambia AND Zimbabwe OR Zimbabwe Rhodesia OR Southern Rhodesia OR Republic of Zimbabwe OR Rhodesia, Southern AND Africa, Northern OR Northern Africa OR North Africa OR Maghreb OR Maghrib OR Sahara AND Algeria AND Egypt OR Arab Republic of Egypt OR United Arab Republic AND Libya AND Morocco OR Ifni AND Tunisia AND Africa, Western OR Africa, West OR West Africa OR Western Africa AND Benin OR Republic of Benin OR Dahomey AND Burkina Faso OR Upper Volta OR Burkina Fasso AND Cabo Verde OR Republic of Cape Verde OR Cape Verde AND Cote d'Ivoire OR Ivory Coast OR Republic of Cote dilvoire AND Gambia OR Republic of the Gambia AND Ghana OR Republic of Ghana OR Gold Coast AND Guinea OR Guinea, French OR Republic of Guinea OR French Guinea OR Guinea, Republic of AND Guinea-Bissau OR Republic of Guinea-Bissau OR Portuguese Guinea OR Guinea, Portuguese OR Guinea-Bissau, Republic of AND Liberia OR Republic of Liberia AND Mali OR Republic of Mali AND Mauritania AND Niger OR Republic of Niger AND Nigeria OR Federal Republic of Nigeria AND Senegal OR Republic of Senegal AND Sierra Leone OR Republic of Sierra Leone AND Togo OR Togolese Republic] 
Data collection tool (Google Forms)

https://docs.google.com/forms/d/1u7H7SBc7z3pCcZ8vIA87W5CPwYFb9qIG1pu0LWQVtx c/edit 


\section{Functional Neurosurgery in Africa: A scoping review}

In 2015, the Lancet Commission on Global Surgery estimated that 143 million additional surgical procedures are needed in low- and middle-income countries each year, and a subsequent study revealed that approximately $15 \%$

of those surgical procedures are neurosurgical. Moreover, the dearth of neurosurgeons particularly in Africa where a discrepancy in their distribution between urban and suburban areas, tend to enlarge the gap. In our study we set a particular interest in the surgical management of movement disorders, epilepsy and pain, through functional neurosurgery (FN). FN is a set of procedures that involves precise surgical targeting of anatomic structures to modulate or improve a neurologic function. In Africa, FN still remains a luxury tool, where only few countries

*Obligatoire

1. Authors * 
2. Country *

Une seule réponse possible.

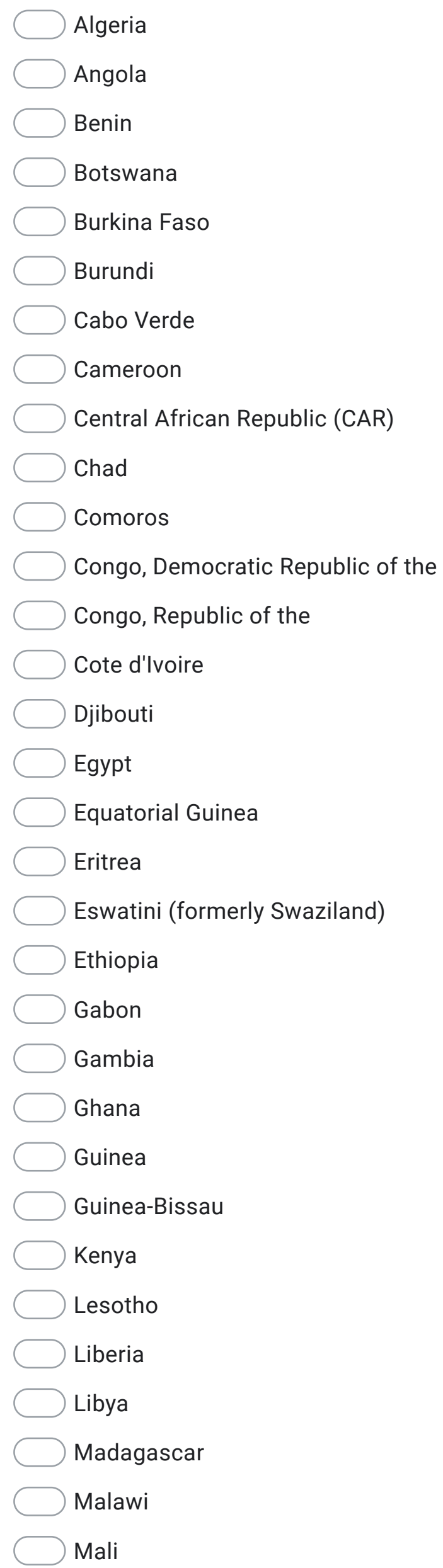




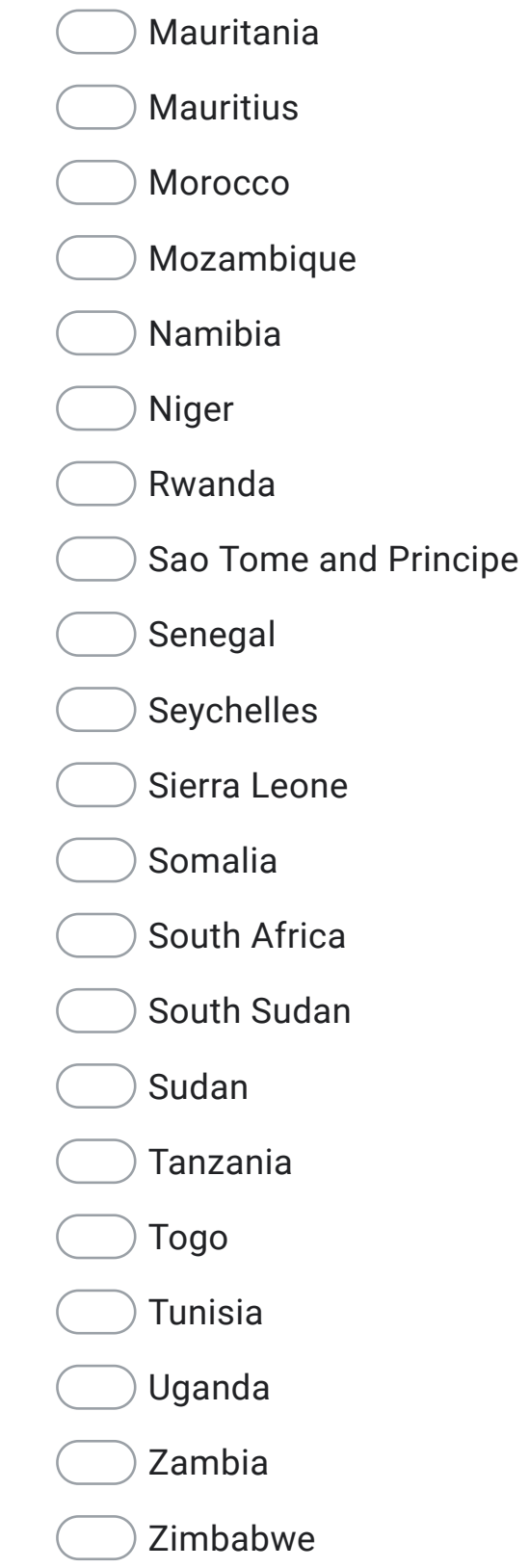


3. Publication year *

Une seule réponse possible.

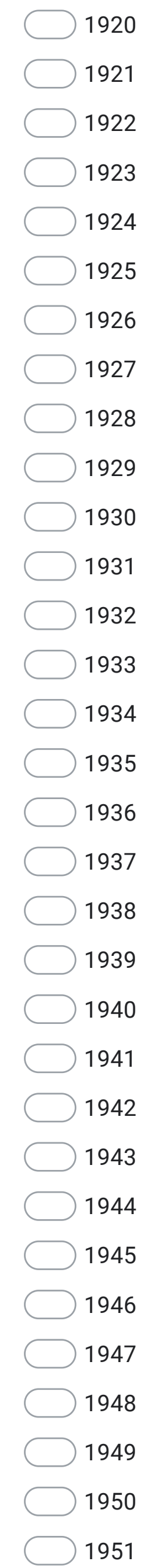


1952

1953

1954

1955

$\longrightarrow 1956$

1957

1958

1959

1960

1961

1962

$\longrightarrow 1963$

1964

1965

1966

1967

1968

1969

$\longrightarrow 1970$

1971

1972

1973

1974

$\longrightarrow 1975$

1976

1977

1978

1979

1980

1981

1982

1983

1984

1985

1986 


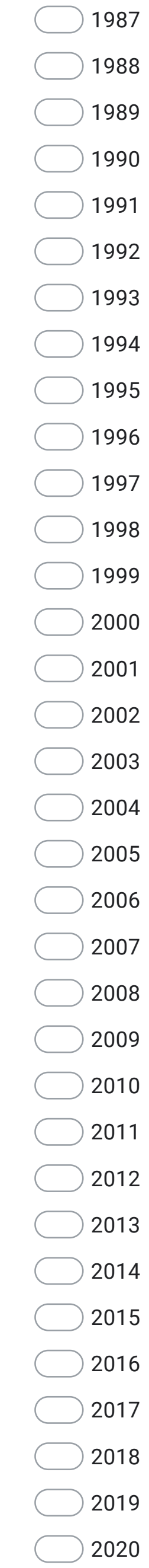


4. Publication type *

Une seule réponse possible.

Adaptive Clinical Trial

$\bigcirc$ Address

Autobiography

$\longrightarrow$ Bibliography

Biography

Case Report

$\bigcirc$ Case Series

Classical Article

Clinical Conference

Cohort Study

Commentary

Congress

Cross Sectional Study

Duplicate Publication

Editorial

Evaluation Studies

Government Document

Guideline

$\bigcirc$ Historical Article

Introductory Journal Article

Letter to the Editor

Literature Review

Meta-Analysis

Multicenter Study

Newspaper Article

Observational Study

Periodical Index

Personal Narrative

Portrait

Practice Guideline

Review

Scientific Integrity Review 
Study Characteristics

Support of Research

Systematic Review

Technical Report

$\bigcirc$ Validation Studies

Other

\section{Type of Hospital *}

Une seule réponse possible.

$\bigcirc$ Public

Private

$\bigcirc$ University affiliated

Faith-based

6. Is there a FN training center affiliated *

Plusieurs réponses possibles.

$\square$ yes

$\square$ no

7. if yes, what type of training? *

Une seule réponse possible.

cadaver lab

$\bigcirc$ virtual/simulations

Hands on 
8. How many consultant Functional neurosurgeon present? *

Une seule réponse possible.

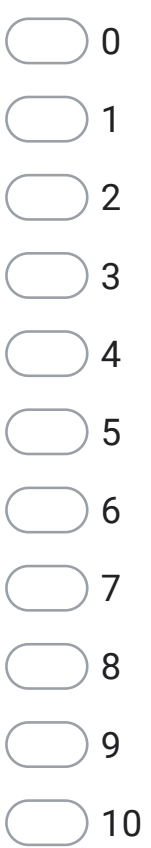

9. Disease *

Plusieurs réponses possibles.

$\square$ Intractable epilepsy

$\square$ Parkinson disease

Chorea and athethosis

$\square$ Pain

$\square$ Psychiatric disorder

Autre :

10. Surgical technique * 
11. Modalities *

Une seule réponse possible.

Neuronavigation

Stereotaxis

Microsurgery

Autre :

12. Difficulties *

Ce contenu n'est ni rédigé, ni cautionné par Google.

Google Forms 